\title{
Pure laparoscopic right hemihepatectomy via anterior approach
}

\author{
Chen Huanwei $^{1} \cdot$ Deng Feiwen ${ }^{1}$
}

Received: 19 September 2015/Accepted: 2 April 2016/Published online: 29 April 2016

(C) Springer Science+Business Media New York 2016

\begin{abstract}
Background In open hepatectomy ear, right hemihepatectomy via anterior approach has been accepted as one of the standard methods in some specialized centers. However, pure laparoscopic right hemihepatectomy by anterior approach is technically demanding [1-5]. Here, we described the technique of pure laparoscopic right hemihepatectomy via anterior approach.

Methods We performed three pure laparoscopic right hemihepatectomies via anterior approach between 2013 and 2014. This video illustrated this procedure steps in a 32 years old male with hepatocellular carcinoma. Right hemihepatectomy was performed after hemihepatic vascular inflow occlusion using the lowering of the hilar plate approach [6]. The hepatic parenchyma transection via anterior approach was performed with Ligasure, ultrasound scalpel and bipolar electrocoagulation. The right hepatic vein was divided with an endoscopic vascular stapler. Thereafter, mobilization of the right liver was performed. Results The operation time was $240 \mathrm{~min}$, and the blood loss was $150 \mathrm{ml}$. The recovery was uneventful and the patient discharged on postoperative day 8 . The pathology was hepatocellular carcinoma with vascular invasion and
\end{abstract}

Electronic supplementary material The online version of this article (doi:10.1007/s00464-016-4903-0) contains supplementary material, which is available to authorized users.

Chen Huanwei

Chwei_fsyyy@163.com

1 The Liver and Pancreatic Department, The Affiliated Foshan Hospital, Sun Yat-Sen University, The Lingnan Road NO.2, Chancheng District, Foshan 528000, Guangdong Province, China also satellites foci adjacent to the main tumor. The resection margin was negative.

Conclusion Pure laparoscopic right hemihepatectomy via anterior approach was safe and feasible.

Keywords Endoscopy $\cdot$ Hepatic cancer $\cdot$ Surgical technique

\section{Compliance with ethical standards}

Disclosures Chen Huanwei and Deng Feiwen have no conflict of interests or financial ties to disclose.

\section{References}

1. Nomi T, Fuks D, Agrawal A, Kawaguchi Y, Ogiso S, Gayet B (2015) Totally laparoscopic right hepatectomy combined with resection of the inferior vena cava by anterior approach. Ann Surg Oncol 22(3):851

2. Takahashi M, Wakabayashi G, Nitta H, Takeda D, Hasegawa Y, Takahara T, Ito N (2013) Pure laparoscopic right hepatectomy by anterior approach with hanging maneuver for large intrahepatic cholangiocarcinoma. Surg Endosc 27(12):4732-4733

3. Liu L, Zeng Y, Lai W, Diao F, Wu H, Wang J, Chu Z (2014) Laparoscopic anterior approach of major hepatectomy combined with colorectal resection for synchronous colorectal liver metastases. Surg Laparosc Endosc Percutan Tech 24(6):e237-e240

4. Machado MA, Surjan RC, Makdissi FF (2011) Video: intrahepatic Glissonian approach for pure laparoscopic right hemihepatectomy. Surg Endosc 25(12):3930-3933

5. Ho CM, Wakabayashi G, Nitta H, Takahashi M, Takahara T, Ito N, Hasegawa Y (2013) Total laparoscopic limited anatomical resection for centrally located hepatocellular carcinoma in cirrhotic liver. Surg Endosc 27(5):1820-1825

6. Chen H, Wang F, Deng F, Zhen Z, Lai EC, Lau WY (2014) Laparoscopic right hemihepatic vascular inflow occlusion by lowering of the hilar plate. J Laparoendosc Adv Surg Tech A. 24(12):833-836 